\title{
Neuronal Ceroid Lipofuscinosis: A Common Pathway?
}

\author{
DIXIE-ANN PERSAUD-SAWIN, TALAL MOUSALLEM, CHRISTINE WANG, ADAM ZUCKER, EIKI KOMINAMI, \\ AND ROSE-MARY N. BOUSTANY
}

\begin{abstract}
Department of Pediatrics [D.-A.P.-S., T.M., C.W., A.Z., R.-M.N.B.], Department of Neurobiology [R.-M.N.B.] Duke University Medical Center, Durham, North Carolina, 27710; Laboratory of Neurobiology/Neurotoxicology [D.-A.P.-S., ], NIEHS/NIH, RTP, North Carolina, 27709; Department of Biochemistry [E.K.], Juntendo University School of Medicine, Tokyo 113-8421, Japan; Abu-Haidar Neuroscience Institute [R.-M.N.B.], American University of Beirut Medical Center, Beirut 1107 2020, Lebanon
\end{abstract}

\begin{abstract}
The neuronal ceroid lipofuscinoses are pediatric neurodegenerative diseases with common clinical features. Of the nine clinical variants (CLN1-CLN9), six have been genetically identified. Most variants manifest cell death and dysregulated sphingolipid metabolism, suggesting the proteins defective in these disorders may interact along one pathway. NCL patientderived cell lines exhibit cell growth and apoptotic defects that reverse following transfection with the wild-type gene. The membrane-bound proteins CLN3, CLN6, and CLN8 complement each other, as do CLN1 and CLN2 proteins, with respect to growth and apoptosis. The CLN2 protein also corrects growth and apoptosis in CLN3-, CLN6-, and CLN8-deficient cell lines. Neither CLN1deficient nor CLN2-deficient growth defects are corrected by CLN3, CLN6, and CLN8 proteins. CLN2, CLN3, CLN6, and CLN8 proteins co-immunoprecipitate and co-localize to early and/or recycling endosomes and lipid rafts. Additionally, CLN2p and CLN1p co-immunoprecipitate. The work presented supports interactions between NCL proteins occurring at multiple points along one pathway. (Pediatr Res 61: 146-152, 2007)
\end{abstract}

$\mathrm{T}$ he NCLs (neuronal ceroid lipofuscinosis) are pediatric neurodegenerative disorders. The nine clinical variants are caused by mutations in different genes (CLN1-CLN9). Six of the genes are identified: CLN1, CLN2, CLN3, CLN5, CLN6, and CLN8. These diseases manifest blindness, seizures, cognitive and motor decline, and early death. There is massive neuronal death in the brain.

INCL (CLN1) sets in before age $2 \mathrm{y}$. LINCL begins at $1-4$ $\mathrm{y}$ and has mutations in the CLN2 gene. Mutations in CLN3 result in JNCL. Symptoms begin between 4 and $10 \mathrm{y}$. The gene for adult NCL (CLN4, or Kufs disease) is unknown. Variant forms of LINCL (vLINCL) include the following: Finnish variant, with mutations in the CLN5 gene begins at 4-7 y; Portuguese/Costa Rican variant/CLN6 gene, which presents between $18 \mathrm{mo}$ and $8 \mathrm{y}$; Turkish variant, which manifests between 3 and $7 \mathrm{y}$, and progressive epilepsy with mental retardation (EPMR), which manifests between ages 5

Received August 11, 2006; accepted October 6, 2006.

Correspondence: Rose-Mary N. Boustany, M.D., Department of Pediatrics, Research Drive, MSRB 281B, Duke University Medical Center, Durham, NC, 27710; e-mail: boust001@mc.duke.edu

DOI: $10.1203 / p d r .0 b 013 e 31802 d 8 a 4 a$ and $10 \mathrm{y}$ and has mutations in the CLN8 gene. Some Turkish vLINCL cases have mutations in the CLN8 gene.

A new NCL variant, CLN9, is clinically similar to JNCL. The gene remains unidentified. Fibroblasts from these patients grow at accelerated rates and have increased apoptosis and low ceramide and sphingomyelin levels.

CLN1 and CLN2 code for the enzymes PPT1 (palmityl protein thioesterase 1) and TPP1 (tripeptidyl peptidase 1), respectively. PPT1, a glycoprotein (34 kD), removes longchain fatty acids from cysteine. Its deficiency leads to accumulation of long-chain fatty acid cysteine thioesters. TPP1 (48 $\mathrm{kD}$ ) is a lysosomal, serine carboxyl peptidase that removes tri-peptides from oligopeptides.

CLN3, CLN6, and CLN8 are membrane-bound proteins. The CLN5 protein is a membrane glycoprotein, and the CLN6 protein (36 kD monomer, $60 \mathrm{kD}$ dimer) localizes to endoplasmic reticulum [for review see (1)]. Wild-type CLN3 protein (CLN3p, 48-52 kD) traffics from Golgi to lipid rafts at the plasma membrane via Rab4- and Rab11-positive endosomes. CLN3-deficient cells undergo apoptotic/caspase-mediated autophagic cell death (2). CLN3p is neuroprotective and harbors a galactosylceramide (GalCer) lipid raft binding domain suggesting that it may be a GalCer transporter (3).

CLN8 belongs to the TLC family (Tram, Lag1 and CLN8) and may impact dihydroceramide synthase (4). CLN8 protein $(33 \mathrm{kD})$ localizes to the ER/ER-Golgi intermediate compartment (ERGIC). The mouse homolog shares $82-85 \%$ sequence identity with its human counterpart and the mnd mouse is a naturally occurring animal model (1).

CLN3-deficient cells have high levels of ceramide/ sphingomyelin that are corrected by CLN3 $(5,6)$. CLN9-deficient cells have diminished ceramide/sphingomyelin. Wildtype CLN9 is thought to be an activator of dihydroceramide synthase, the enzyme generating dihydroceramide, a ceramide precursor (7).

Sphingolipids impact cell death, growth, and differentiation and are components of lipid rafts, specialized platforms that orchestrate signaling events (8-13). They are implicated in the pathogenesis of neurodegenerative diseases such as Alzheimer's and prion diseases (14).

Abbreviations: CLN1-9, CLN1-9 protein; GalCer, galactosylceramide; INCL, infantile-onset NCL; JNCL, juvenile-onset NCL; LINCL, late infantile-onset NCL; NCL, neuronal ceroid lipofuscinosis 
NCL protein deficiencies result in similar cell biologic phenotypes including dysregulated cell growth, apoptosis, and abnormal sphingolipid/phospholipid levels in CLN1-, CLN2-, CLN3-, CLN6-, and CLN8-deficient cells and neurodegeneration (1). We investigate whether NCL proteins may interact at multiple points along a common pathway.

\section{METHODS}

Cell lines. Fibroblast/immortalized lymphoblast cell lines, derived from normal controls/patients with defects in CLN1, CLN2, CLN3 (JNCL), or CLN6 genes, were used. Cells from the mnd-I- (mouse cln8) knockout mouse and its control C57B6 were obtained from the Jackson Labs (Bar Harbor, ME). Cos-7 fibroblasts (ATCC, Manassas, VA) were also used. CLN1deficient cells were kindly provided by Dr. Sandra Hoffman. Cell line mutations were CLN1-homozygous R151X, CLN2-homozygous 3556G > C, CLN3-homozygous c.46-677del, and CLN6-homozygous G317G. Use of patient cells is covered by a Duke University Institutional Review Boardapproved protocol.

Tissue culture. Cos-7 and human fibroblasts were grown in Dulbecco's modified Eagle's medium (DMEM) with 10\% fetal bovine serum (FBS), lymphoblasts in RPMI $164010 \%$ FBS, (Invitrogen, Carlsbad, CA) at $37^{\circ} \mathrm{C}$ and $5 \% \mathrm{CO}_{2}$. Mouse cell lines were grown in $20 \%$ horse serum.

Transfection. cDNA for, CLN1, CLN2, CLN3, CLN6, and CLN8 were cloned into pGEM(7+) vector and transfected into cells (2). Fibroblasts were plated at a density of $1-5 \times 10^{4}$ cells $/ 0.44 \mathrm{~cm}^{2}$. Transfection efficiency was assessed with pGEM-YFP fluorescent vector, and was $60-70 \%$ for fibroblasts and $80-90 \%$ for lymphoblasts $(p<0.05)$.

JC-1 staining and trypan blue dye exclusion assay. Staining with the mitochondrial membrane potential dye, JC-1, and the trypan blue assay are previously described (2). Statistical significance was determined using the $t$ test.

Propidium iodide staining. Cells were centrifuged, washed, and resuspended in $100 \mu \mathrm{L}$ PBS, placed on a glass slide, then incubated with $100 \mu \mathrm{L}$ $0.5 \mathrm{mg} / \mathrm{mL}$ propidium iodide solution, washed, and viewed using a Leica fluorescent microscope at $20 \times$ magnification. Three hundred cells from three fields of vision were counted. The $t$ test was applied.

Co-immunoprecipitation and Western blotting. Cells, 1.5-2 $\times 10^{6}$, were snap-frozen at $-80^{\circ} \mathrm{C}$, thawed, and resuspended in $200-300 \mu \mathrm{L}$ lysis buffer [250 $\mu \mathrm{L}$ proteinase cocktail inhibitor (Sigma Chemical Co.-Aldrich, St. Louis, MO), $4.7 \mathrm{~mL}$ Freedman Buffer, $50 \mu \mathrm{L}$ phenylmethylsulphonylfluoride (PMSF)] and centrifuged at $13,000 \mathrm{rpm}$ at $4^{\circ} \mathrm{C}$. The same amount of cells was used for each immunoprecipitation. The total protein concentration of each sample was determined by the Bradford Assay (Bio-Rad, Hercules, CA) before immunoprecipitation ensuring that protein concentration was the same for each immunoprecipitation. As the no- immunoprecipitation control, 1/10 vol of the supernatant or cell lysate was reserved. Five micrograms of antibody was added to the rest of the lysate and tumbled at $4^{\circ} \mathrm{C}$ for $2 \mathrm{~h}$. Twelve microliters of IgG agarose beads (Santa Cruz Biotechnologies, Santa Cruz, CA) added, and tumbled at $4^{\circ} \mathrm{C}$ for $1-2 \mathrm{~h}$. The mix was washed three times with lysis buffer at $4^{\circ} \mathrm{C}$. Twenty-four microliters $2 \times$ dissociation buffer was added, boiled for $8 \mathrm{~min}$, cooled, and loaded onto a $4-20 \%$ ready gel (Bio-Rad). The membrane was blocked at room temperature (RT) in 5\% nonfat dry milk/PBST $/ 5 \%$ goat serum $/ 1 \%$ BSA $/ 1 \times \mathrm{PBS} / 2.5 \%$ BSA in TBST, depending on the protein detected. Antibody was added at 1:1000 dilution and incubated at $4{ }^{\circ} \mathrm{C}$ overnight. The blot was washed with PBS/Tween and incubated with the appropriate secondary antibody at 1:12,000 (CLN1, CLN2, and CLN3) or 1:14,000 (CLN6 and CLN8) dilution in block for $1 \mathrm{~h}$ at room temperature. Membrane was washed and developed with ECLplus (Amersham Pharmacia Biotech, Inc., Piscataway, NJ).

Controls. For newly made antibodies, CLN6 and CLN8 total protein was probed with presera from either antibody to ensure absence of contaminating proteins or nonspecificity. Blots were probed with antibodies to proteins that do not bind CLN proteins. For CLN antibodies made in the same host species or similar in size, the membrane used for co-IP was probed once. Antibodies were presorbed on separate membranes.

Antibodies. Antibodies included previously characterized rabbit polyclonal anti-CLN3 antibody $(3,4)$, sheep polyclonal anti-CLN6 antibody made to residues 284-301 of the CLN6 protein, sheep polyclonal anti-CLN8 antibody made to residues 2-19 of the CLN8 protein, rabbit polyclonal anti-CLN2 antibody (Orbigen Inc., San Diego, CA), rabbit polyclonal antiCLN1 antibody (gift from Dr. Sandra Hoffman), rat polyclonal antiGRASP65 antibody (gift from Dr. Francis Barr), goat polyclonal anti-alkaline phosphatase antibody, goat polyclonal anti-cathepsin D antibody, mouse monoclonal anti-Rab11 antibody, and mouse monoclonal anti-Rab4 antibody (Research Diagnostics Inc., Concord, MA). Alexa Fluor 488 and 543 secondary antibodies were used (Molecular Probes, Eugene, OR).

Co-localization studies. Cells were plated, fixed, and stained as previously described (4). Primary and secondary Alexa Fluor antibodies were added at a 1:1000 dilution and incubated at RT. Cells were imaged with a Zeiss Laser Scanning Confocal Microscope 510 (Carl Zeiss Inc., Thornwood, NY) in 40 Z-planes every $0.1 \mu \mathrm{m}$ from top to bottom of the cell with a $100 \times$ oil objective. Planes with maximal co-localization were used. Cells from six randomly chosen fields of vision were counted and protein distribution patterns documented. Each experiment was repeated with "no-antibody" controls.

\section{RESULTS}

NCL variants show defective cell growth and increased apoptosis. CLN3-deficient (JNCL) cells have slowed cell growth and accelerated apoptosis (2). CLN1-, CLN2-, and CLN6-deficient fibroblasts, and mnd-/- mouse fibroblasts are analyzed in this study. CLN1-, CLN2-, CLN3-, and CLN6deficient cells have slowed growth (Fig. 1, $A$ and $B$ ). CLN8deficient mouse fibroblasts show increased growth (Fig. 1C).

JC-1 and propidium iodide staining demonstrated that all CLN-deficient lymphoblast or fibroblast (mnd-I-) cell lines exhibited increased apoptosis and support the growth analyses $(p<0.05)$. NCL-derived cells show increased etoposideinduced apoptosis. For mouse fibroblasts, $0.25 \mu \mathrm{g} / \mu \mathrm{L}$ and, for human fibroblasts or lymphoblasts, $1 \mu \mathrm{g} / \mu \mathrm{L}$ caused $50 \%$ of cells to die.

Correction of cell growth and apoptosis. Cells were transfected with a vector control (EV) or with CLN constructs. CLN3 cDNA added to JNCL cells resulted in $48 \%$ protection from etoposide-induced apoptosis $(p<0.05)$, supporting pre-
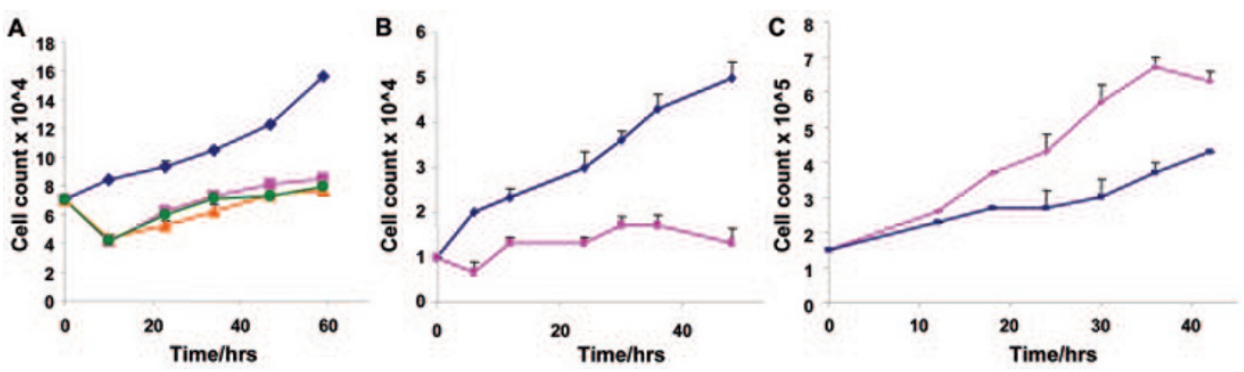

Figure 1. Growth of normal and CLN-deficient cell lines. Growth curves of (A) CLN1-, CLN2-, and CLN3-deficient lymphoblasts [wild-type (blue); CLN3 (pink); CLN1 (orange); CLN2 (teal)]. (B) Wild-type (blue) and CLN6-deficient lymphoblasts (pink). (C) mnd-/- (pink) and normal (blue) mouse fibroblasts. Cell lines were also transfected with a vector control. Each data point represents average of three replicates for $n=3$ experiments. Time points after $18 \mathrm{~h}$ have a $p$ value $<0.05$. 
vious work (2). Introduction of CLN2 cDNA into LINCL cells afforded 58\% protection from etoposide $(p<0.05)$. Transfection of INCL cells with CLN1 provided $38 \%$ protection from etoposide $(p<0.05)$. CLN6 cDNA corrected apoptosis and provided $40 \%$ protection from etoposide-induced apoptosis in CLN6-deficient cells $(p<0.05)$. Transfection of mnd-/- cells with human CLN8 cDNA provided $80 \%$ protection from apoptosis induced by etoposide. Growth and apoptotic defects are corrected by the intact cDNA, and human CLN8 and the mouse cln8 gene are functionally homologous.

Correction of growth and apoptosis in CLN1- and CLN2deficient cells. Cell growth and apoptotic defects in CLN2deficient cells were corrected by CLN1 or CLN2 cDNA. The introduction of CLN1 cDNA provided cells with 54\% protection from etoposide-induced apoptosis $(p<0.05)$. Introduction of other NCL genes had no effect.

Growth and apoptosis in CLN1-deficient cells were corrected by CLN1 or CLN2 cDNA only. The introduction of CLN2 cDNA provided $22-35 \%$ protection from etoposide-induced apoptosis $(p<0.05)$. Other NCL genes had no effect $(p>0.05)$.

Correction of cell growth and apoptotic defects in CLN3and CLN6-deficient cells. CLN2 cDNA resulted in partial correction of growth and apoptotic defects in CLN3-deficient cells. Protection from apoptosis was $24.4-26 \%(p<0.05)$. Introduction of CLN6 cDNA completely restored cell growth in CLN3-deficient cells, and provided JNCL cells with 46$48 \%$ protection from etoposide-induced apoptosis $(p<0.005$; Fig. 2A). Transfection of CLN3-deficient (JNCL) patient cells with CLN8 cDNA also resulted in near total correction of the growth defect, similar to transfection with CLN3 cDNA ( $p>$ 0.05). CLN1 had no effect on growth or apoptosis of JNCL cells. This suggests that CLN2, CLN3, CLN6, and CLN8 proteins may function in one pathway, or may interact. Protection from etoposide-induced apoptosis was assessed using JC-1/PI staining.

CLN2 cDNA added to CLN6-deficient cells corrected growth and provided 33\% protection from etoposide-induced apoptosis. CLN3 or CLN8 cDNA significantly reversed growth defects (Fig. $2 B$ ) and provided cells with $42 \%$ and $39 \%$ protection from etoposide-induced apoptosis, respectively. CLN1 cDNA had no effect on their growth or death.

Correction of cell growth and apoptotic defects in mnd-/cells. Accelerated cell growth in CLN8-deficient mouse fibroblasts (mnd-/-) was restored to normal by CLN2 (not shown), CLN3, and CLN6 cDNA (Fig. 2C). CLN2, CLN3, or CLN6 cDNA provided mnd-I- mouse fibroblasts with $80 \%$ protec-
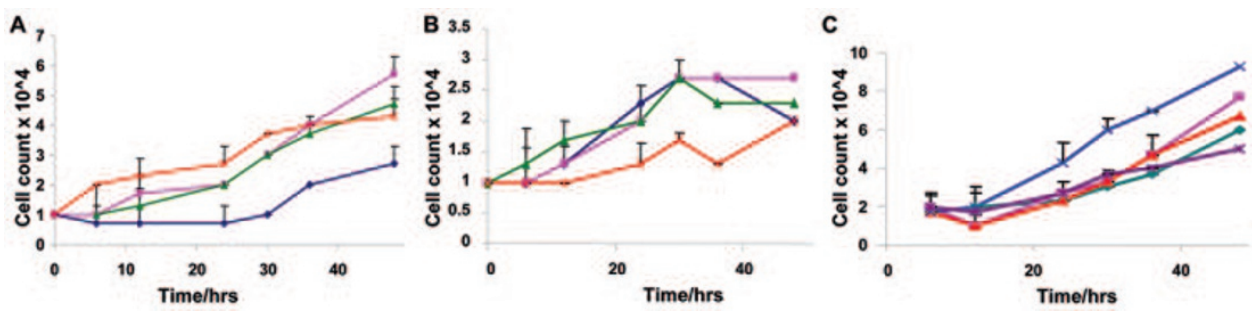

Figure 2. Correction of growth defects of CLN3-, CLN6-, and CLN8-deficient cell lines. (A) Growth curves of CLN3-deficient (JNCL) lymphoblasts by CLN3 (pink), CLN6 (red), and CLN8 (green) cDNA and vector control (blue) $(p<0.05)$. (B) Growth curves of vLINCL (CLN6-deficient) lymphoblasts with CLN3 (blue), CLN6 (pink), and CLN8 (green) cDNA and vector control (red $)(p<0.05)$. (C) Growth curves of mnd-/- (CLN8-deficient) mouse fibroblasts transfected with CLN3 (teal), CLN6 (pink), and CLN8 (red) cDNA and vector control (blue) $(p<0.05)$ compared with wild-type C57B6 mouse fibroblasts (purple). Each point represents average of three replicates for $n=3$ experiments.

A
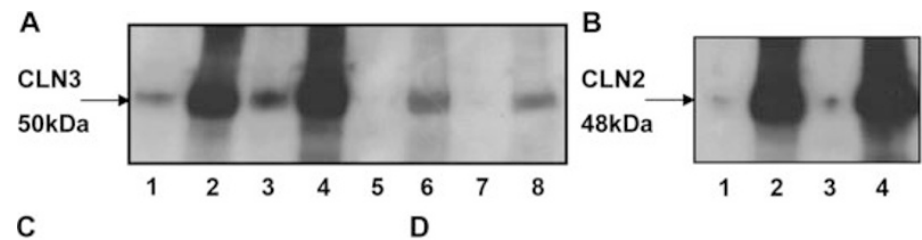

C

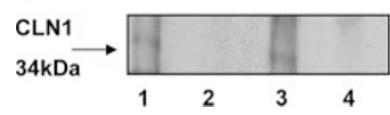

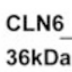
$36 \mathrm{kDa}$

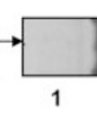

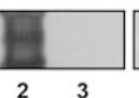
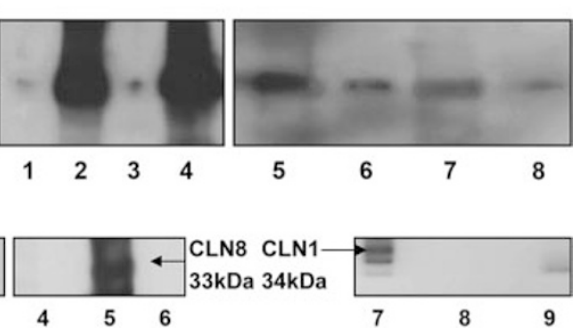

Figure 3. Immunoprecipitation with CLN antibodies. (A) Western blot of lysates from normal lymphoblasts following immunoprecipitation with CLN2, CLN3, CLN6, and CLN8 antibodies and probing with CLN3 antibody. Lanes 1, 3, 5, and 7: control lysate before immunoprecipitation; lane 2: lysate following immunoprecipitation with CLN2; lane 4: lysate following immunoprecipitation with CLN3; lane 6: lysate following immunoprecipitation with CLN6; lane 8: lysate following immunoprecipitation with CLN8. One hundred micrograms of protein per control lane. (B) Western blot (normal lymphoblasts) following immunoprecipitation with CLN2, CLN3, CLN6, and CLN8 antibody and probing with CLN2 antibody. Lanes 1, 3, 6, and 8: control lysate before immunoprecipitation; lane 2: lysate following immunoprecipitation with CLN2; lane 4: lysate following co- immunoprecipitation with CLN3; lane 5: lysate following immunoprecipitation with CLN6; lane 7: lysate following immunoprecipitation with CLN8. (C) Western blot: Cos-7 cells following immunoprecipitation with the CLN1/CLN2 antibody and probed with anti-CLN1 antibody. Lane 1: cell lysate was immunoprecipitated with CLN1 antibody; lanes 2 and 4: control lysate before immunoprecipitation; lane 3: lysate was immunoprecipitated with CLN2 antibody. (D) Western blot analysis (cos-7 cells) following immunoprecipitation with CLN1, CLN6, and CLN8 antibodies. Lane 1: cell lysate was immunoprecipitated with CLN1 antibody and probed with CLN6; lane 2: lysate was immunoprecipitated with CLN6 and probed with CLN6; lanes 3, 6, and 9: control lysate before immunoprecipitation; lane 4: lysate was immunoprecipitated with CLN1 and probed with CLN8 antibody; lane 5: lysate immunoprecipitated with CLN8 and probed with CLN8; lane 7: lysate following immunoprecipitation with CLN1 and detection with an anti-CLN1 antibody. Lane 8: lysate following immunoprecipitation with CLN3 and detection with an anti-CLN1 antibody. In control lanes, $100 \mu \mathrm{g}$ of protein were loaded. Experiments were repeated twice. 
tion from etoposide-induced apoptosis. CLN1 corrected neither growth nor apoptotic defects in mnd-/- cells.

CLN2, CLN3, CLN6, and CLN8 Co-immunoprecipitate. Normal human lymphoblasts/cos-7 cells were used for immunoprecipitation experiments as they provide a large amount of protein for Western blot. Total protein from these cells was prepared by lysing cells with detergent, as described in "methods." The cell lysate from lymphoblasts was immunoprecipitated with an anti-CLN2 antibody and probed with an antibody to CLN3. A double-band at $48-50 \mathrm{kD}$, corresponding to the CLN3 protein was detected (Fig. 3A, lanes 2 and 4). Control lanes show cell lysate before immunoprecipitation (lanes 1 and 3) with $100 \mu \mathrm{g}$ total protein. Cell lysate immunoprecipitated with an anti-CLN3 antibody and probed with an antibody to CLN2, detected a $48-50 \mathrm{kD}$, band, identifying the CLN2 protein (Fig. 3B, lanes land 4). The same concentration of total protein was immunoprecipitated from each cell sample, ensuring that the baseline level of total protein across samples was the same. Separate membrane blots were used to eliminate possible cross-reactivity between antibody and closely sized CLN proteins made in same or similar host species. This suggests that CLN2 and CLN3 proteins may interact.

Western blot analysis of proteins immunoprecipitated with an anti-CLN6/CLN8 antibody, and probed with an antibody to CLN3 identified the CLN3 protein (Fig. 3A, lanes 5-8). In control lanes, endogenous CLN6p and CLN8p were below detection limits. Transfection and immunoprecipitation increase and concentrate proteins, so cos-7 cells were transfected with plasmids containing CLN6 and CLN8 cDNA, before immunoprecipitation, providing better detection. Reciprocal experiments using transfected cos-7 cells supported protein interactions between CLN3, CLN8, and CLN6 proteins. CLN2 protein immunoprecipitated with CLN6 and CLN8 proteins (Fig. 3B, lanes 5-8), suggesting that CLN3, CLN6, and CLN8 membrane proteins interact with each other, and with soluble CLN2 protein.

CLN1p co-immunoprecipitated with CLN2p (Fig. 3C) and not with CLN3, CLN6, and CLN8 proteins (Fig. 3D). Results from reciprocal immunoprecipitation experiments corroborated this. This is consistent with lack of growth complementation by CLN1 cDNA for CLN3-, CLN6-, and CLN8deficient cells.

CLN2 Co-localizes with CLN3. CLN3 and CLN2 show strong co-localization in a vesicular pattern (Fig. 4A). CLN2 co-localizes with alkaline phosphatase to lipid rafts/plasma membrane (Fig. 4B), with GRASP65 to Golgi (Fig. 4C), with cathepsin D to lysosomes (Fig. $4 D$ ) and with Rab11 to recycling endosomes (Fig. 4E). This suggests that CLN3 and CLN2 proteins may interact with each other at multiple subcellular locations, possibly within Golgi, the plasma membrane and/or within recycling endosomes.

CLN6 co-localizes with CLN2 and CLN3. CLN6 colocalizes with CLN2 and CLN3 proteins (Fig. 5, $A$ and $B$ ). Co-localization with CLN3 was punctate in the plane of the plasma membrane. Co-localization of alkaline phosphatase and CLN6 suggests CLN6 co-localization to lipid rafts (Fig. $5 C$ ). Co-localization of CLN6 and CLN2 was observed below
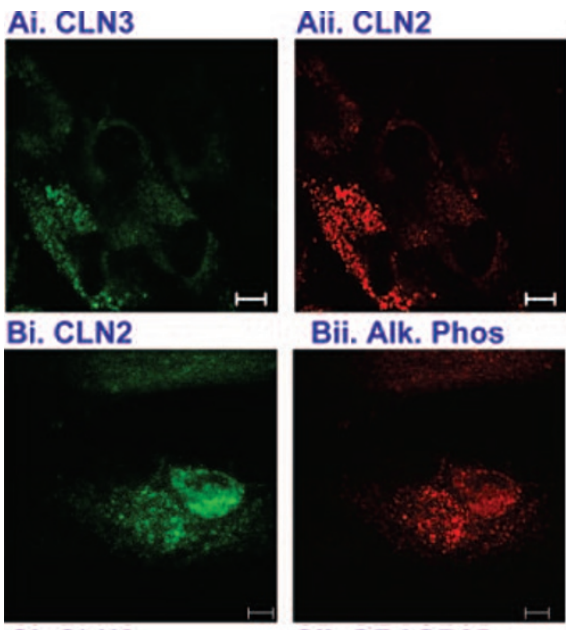

Bii. Alk. Phos
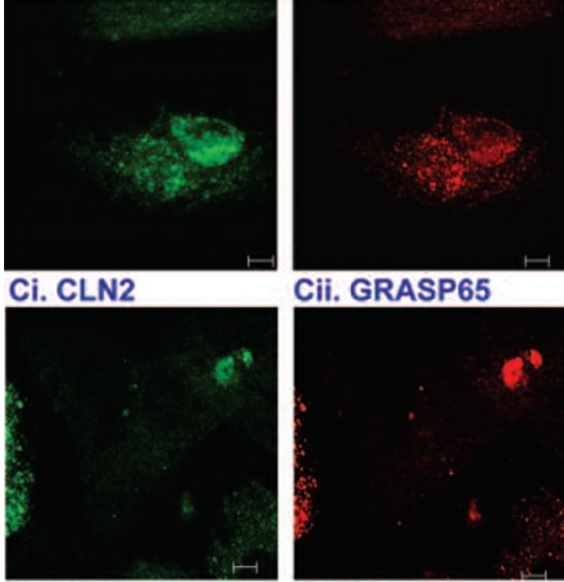

Cii. GRASP65
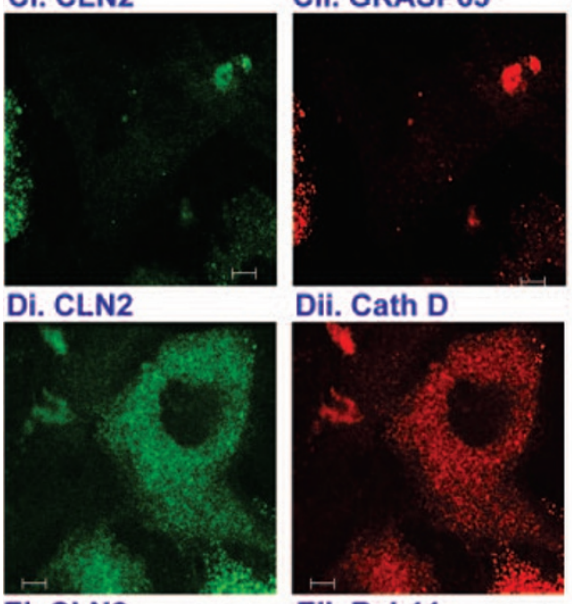

Dii. Cath D
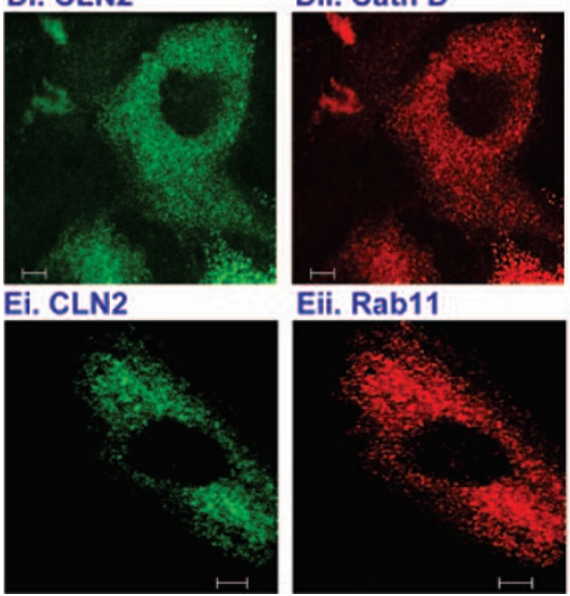

Aiii. Overlay

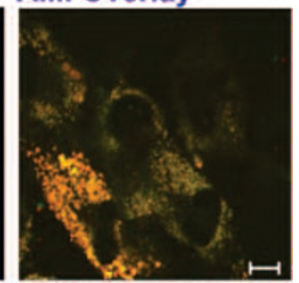

Biii. Overlay

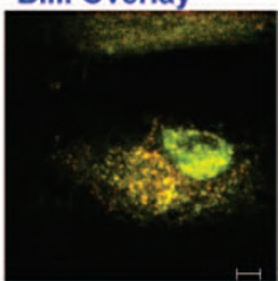

Ciii. Overlay

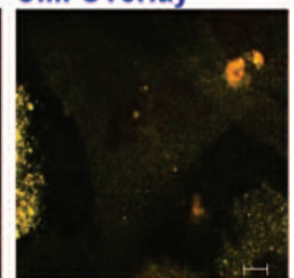

Diii. Overlay

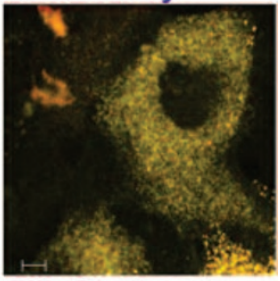

Eiii. Overlay

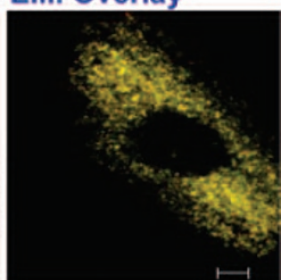

Figure 4. Subcellular localization of CLN2. Normal fibroblasts dually labeled with antibodies to (Ai) CLN3 (green), (Aii) CLN2 (red), (Aiii) overlay. Regions of co-localization are shown in yellow. (Bi) Localization pattern for CLN2 protein (green); (Bii) localization pattern for alkaline phosphatase (red); (Biii) overlay. Regions of co-localization are shown in yellow. (Ci) Localization pattern for CLN2 protein (green); (Cii) localization pattern for GRASP65 (red); (Ciii) overlay. Regions of co-localization are shown in yellow. (Di) Localization pattern for CLN2 protein (green); (Dii) localization pattern for cathepsin D (red); (Diii) overlay. Regions of co-localization are shown in yellow. (Ei) Localization pattern for CLN2 protein (green); (Eii) localization pattern for Rab11 (red); (Eiii) overlay. Regions of co-localization are shown in yellow (magnification, $\times 400$ ). Panels represent specific cellular $\mathrm{Z}$ planes that showed maximal colocalization between the different proteins. Panels are representative of two independent experiments. Scale bars represent $10 \mu \mathrm{M}$.

the plane of the plasma membrane and was vesicular. The distribution pattern was diffuse. CLN6 also co-localized with Rab4 to early endosomes (Fig. 5D). This suggests that the vesicular structures within which CLN6 and CLN2 co-localize are probably early endosomes that recycle to the plasma membrane (Table 1). 


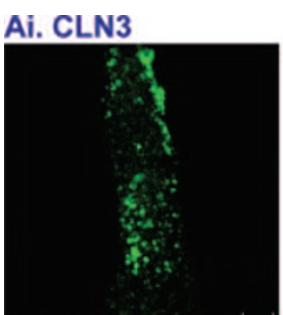

\section{Ci. Alk phos.}

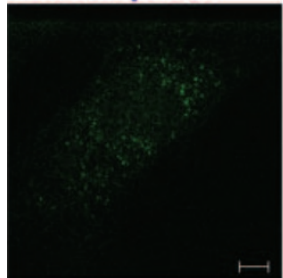

Aii. CLN6

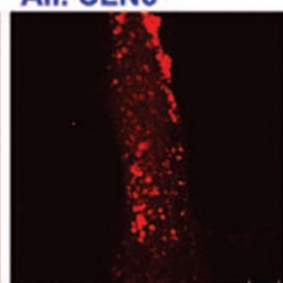

Cii. CLN6

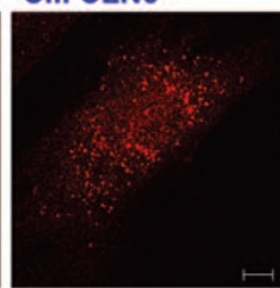

Aiii. Overlay

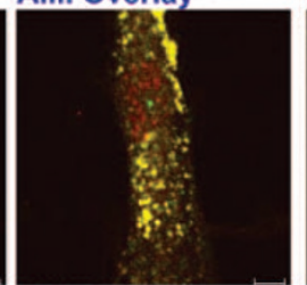

Ciii. Overlay

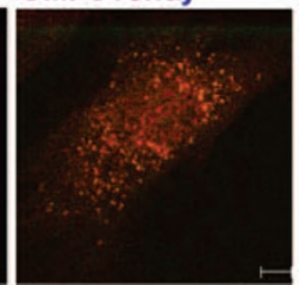

Bi. CLN2

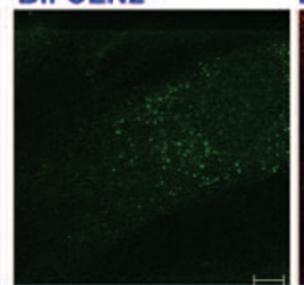

Di. CLN6

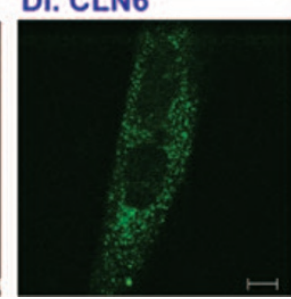

B.ii CLN6

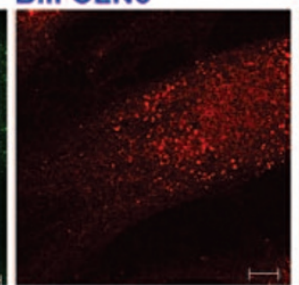

Dii. Rab4

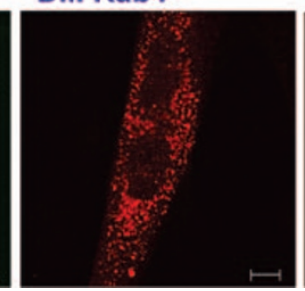

Biii. Overlay

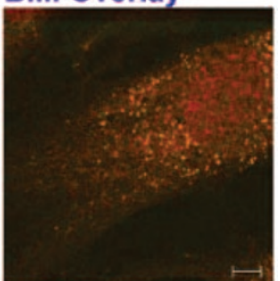

Diii. Overlay

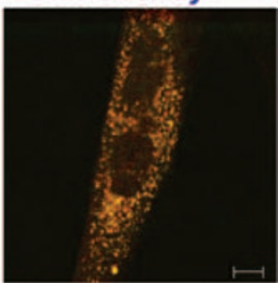

Figure 5. Subcellular localization of CLN6. Normal fibroblasts dually labeled with antibodies to (Ai) CLN3 (green) and (Aii) CLN6 (red); (Aiii) overlay. Regions of co-localization are always shown in yellow. Normal fibroblasts dually labeled with antibodies to (Bi) CLN2 (green) and (Bii) CLN6 (red); (Biii) overlay. Normal fibroblasts dually labeled with antibodies to ( $\mathrm{Ci}$ ) alkaline phosphatase (green) and (Bii) CLN6 (red); (Biii) overlay. Normal fibroblasts dually labeled with antibodies to (Di) CLN6 (green) and (Dii) Rab4 (red); (Diii) overlay (magnification, $\times 400$ ). Panels represent specific cellular Z planes that showed maximal co-localization between the different proteins. Panels are representative of two independent experiments. Scale bars represent $10 \mu \mathrm{M}$.

Table 1. Growth and apoptotic correction of NCL cells

\begin{tabular}{|c|c|c|c|c|c|}
\hline $\begin{array}{l}\text { Cell line } \\
\text { transfected } \\
\text { with }\end{array}$ & CLN1-I- & CLN2-I- & CLN3-I- & CLN6-I- & CLN8-I- \\
\hline CLN1 & $\mathrm{Y}(38 \%)$ & $\mathrm{Y}(54 \%)$ & $\mathrm{N}$ & $\mathrm{N}$ & $\mathrm{N}$ \\
\hline CLN2 & $\mathrm{Y}(35 \%)$ & $\mathrm{Y}(58 \%)$ & $\mathrm{Y}^{*}(24 \%)$ & Y (33\%) & $\mathrm{Y}(80 \%)$ \\
\hline CLN3 & $\mathrm{N}$ & $\mathrm{N}$ & $\mathrm{Y}(48 \%)$ & $\mathrm{Y}(42 \%)$ & $\mathrm{Y}(80 \%)$ \\
\hline CLN6 & $\mathrm{N}$ & $\mathrm{N}$ & $\mathrm{Y}(48 \%)$ & Y $(40 \%)$ & $\mathrm{Y}(80 \%)$ \\
\hline CLN8 & $\mathrm{N}$ & $\mathrm{N}$ & $\mathrm{Y}(37 \%)$ & Y $(39 \%)$ & $\mathrm{Y}(80 \%)$ \\
\hline
\end{tabular}

cDNA from CLN1, CLN2, CLN3, CLN6, and CLN8 genes were transfected into CLN-deficient cells. Cell growth (trypan blue dye exclusion)/ apoptosis-Y, correction of growth/apoptosis, N, no correction. JC-1 assaycells/fields of vision $\times 4$ were counted (dead and alive). The degree of protection, in parentheses (Y panels) equals (percentage of live cells $\ddagger$ treated with etoposide)/(percentage of live cellsł without etoposide) - (percentage of live cells $\dagger$ treated with etoposide)/(percentage of live cells $\dagger$ without etoposide). Each experiment was done in triplicate; $p<0.05$

* Partial correction observed.

$\dagger$ Cells transfected with vector control.

$\ddagger$ Cells transfected with relevant CLN construct.

CLN8 co-localizes with CLN2, CLN3, and CLN6. CLN3 and CLN8 proteins co-localized below the plasma membrane (Fig. 6A). The distribution pattern was punctate. Colocalization between CLN2 and CLN8 was vesicular but less intense than that with CLN3 (Fig. 6B). Co-localization of CLN8 and CLN6 was punctuate, suggesting vesicular structures, and was concentrated at one end of the cell, possibly Golgi or ER (Fig. 6C). CLN8 protein co-localized with alkaline phosphatase to lipid rafts (Fig. $6 D$ ) but was less than that seen with CLN3. These results agree with immunoprecipitation studies and suggest that CLN2, CLN3, CLN6, and CLN8 proteins may traffic between organelles (results summarized in Table 2).

\section{DISCUSSION}

CLN1/CLN2 are lysosomal proteins and CLN3, CLN5, CLN6, and CLN8 are membrane-bound proteins (1). The NCL share clinical manifestations, biochemistry, and pathol- ogy, hence their classification together (5-14). It is probable that all of the NCL genes belong to a common metabolic pathway (15). Precedence for this may be found in the recently proposed association that Niemann-Pick type C (NP-C) and Alzheimer's diseases (AD) may share a common cascade, by which altered cholesterol metabolism leads to neurodegeneration. The involvement of protein complexes in death-receptor and stress-induced apoptosis and neurodegeneration is also well documented $(16,17)$. In this study we explored the possibility that the NCL genes are related by an analogous pathway.

CLN3 cDNA rescues growth and apoptotic defects in CLN3-deficient cells (2). Here, we investigate possible correction of growth defects in NCL cell lines by noncorresponding CLN genes. Growth defects in the NCL cell lines were rescued following transfection with the wild-type gene. Also, CLN3p, CLN6p, and CLN8p complemented each other and interchangeably corrected defects in CLN3- and CLN6deficient/mnd-I- cells. As these are membrane-bound proteins, such complementation suggests that they may interact with each other at a molecular level, possibly forming a functional or structural complex.

CLN2 cDNA complemented growth in CLN3-, CLN6-, and CLN8-deficient cells. This was not reciprocal. This may allude to a downstream position for CLN2 protein in the hypothetical metabolic pathway. Interestingly, correction of defects in CLN3-deficient cells by CLN2 was only partial, in contrast to correction in CLN6-deficient/mnd-/- cells, suggesting CLN2 may be functionally closer to CLN6 and CLN8 than to CLN3.

Previous evidence suggested interactions occur between CLN5, CLN3, and CLN2 proteins (18). Here, CLN3 protein co-immunoprecipitated with CLN6 and CLN8 proteins and CLN6 and CLN8 proteins co-immunoprecipitated with each other. CLN1 interacts with CLN2 but does not bind to CLN3, CLN6, and CLN8 proteins. CLN1 and CLN2 may, therefore, be closely associated (Fig. 7). Co-immunoprecipitation exper- 
Ai. CLN3

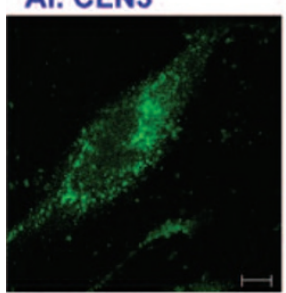

Ci. CLN6

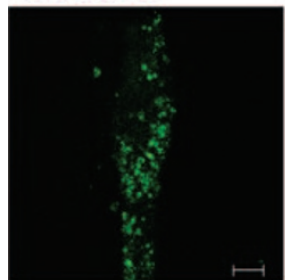

Aii. CLN8

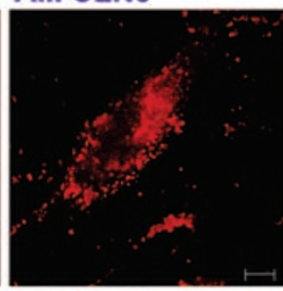

Aiii. Overlay

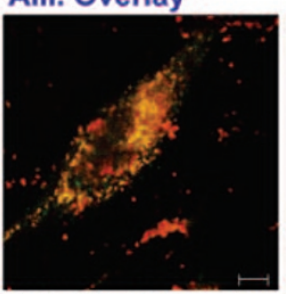

Ciii. Overlay

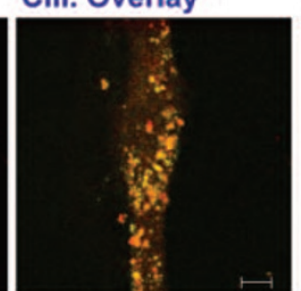

Bi. CLN2

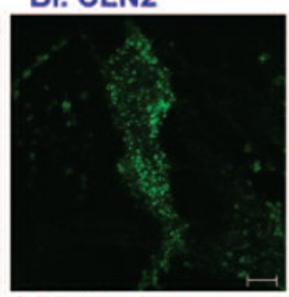

Di. Alk phos

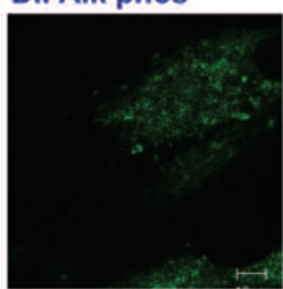

Bii. CLN8

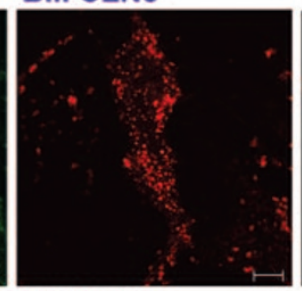

\section{Dii. CLN8}

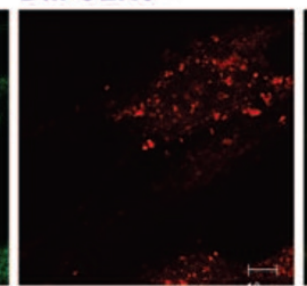

Biii. Overlay

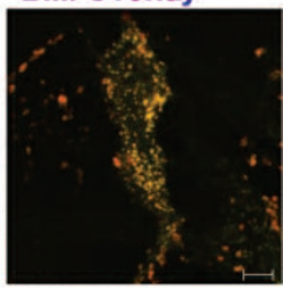

Diii. Overlay

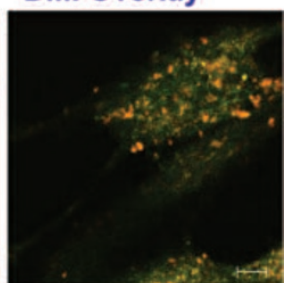

Figure 6. Subcellular localization of CLN8. Normal fibroblasts dually labeled with antibodies to (Ai) CLN3 (green) and (Aii) CLN8 (red); (Aiii) overlay. Regions of co-localization are always yellow. Normal fibroblasts dually labeled with antibodies to (Bi) CLN2 (green) and (Bii) CLN8 (red); (Biii) overlay. Normal fibroblasts dually labeled with antibodies to (Ci) CLN6 (green) and (Cii) CLN8 (red); (Ciii) overlay. Normal fibroblasts dually labeled with antibodies to (Di) alkaline phosphatase (green) and (Dii) CLN8 (red); (Diii) overlay. (Magnification, $\times 400$ ). Panels represent cellular Z planes with maximal colocalization. Panels are representative of two independent experiments. Scale bars represent $10 \mu \mathrm{M}$.

Table 2. Co-localization studies

\begin{tabular}{lllll}
\hline Co-localization with & CLN2 & CLN3 & CLN6 & CLN8 \\
\hline Golgi & Y & Y & Y & Y \\
Lipid rafts & Y & Y & Y & Y \\
Recycling endosomes & Y & Y & Y & Y \\
Lysosomes & Y & N & ND & ND \\
CLN2 & N/A & Y & N & Y \\
CLN3 & Y & N/A & Y & Y \\
CLN6 & Y & Y & N/A & Y \\
CLN8 & Y & Y & Y & N/A \\
\hline
\end{tabular}

Subcellular localization of CLN2, CLN3, CLN6, and CLN8 proteins, and NCL protein co-localization. ND, not done; N/A, not applicable.

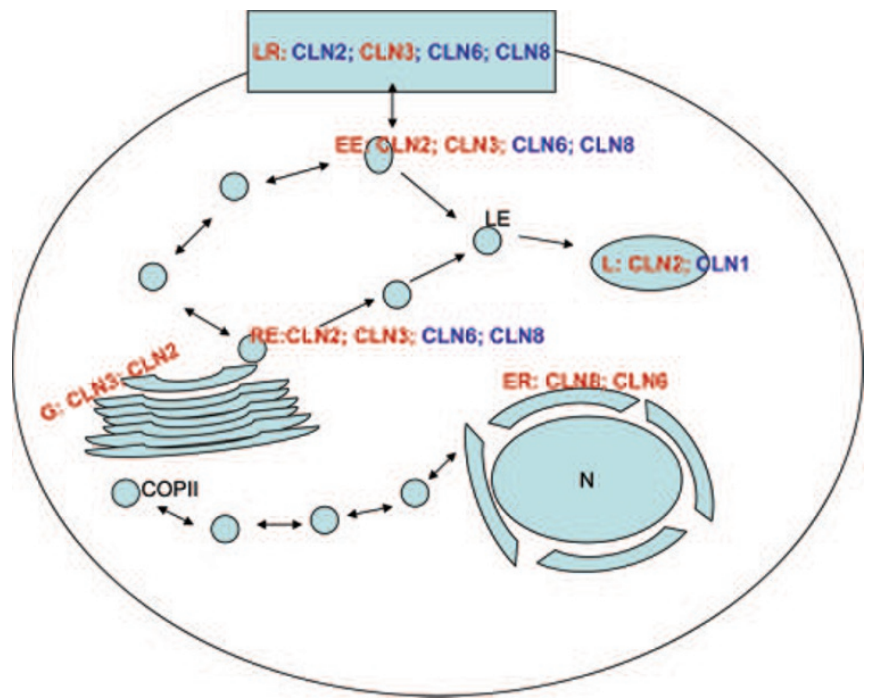

Figure 7. Hypothetical NCL protein interactions. NCL proteins co-localize within subcellular compartments. They recycle from Golgi $(G)$ to lipid rafts $(L R)$ within early $(E E) /$ recycling endosomes $(R E)$. CLN2 within lysosomes $(L)$. Primary locations are identified by red, minor locations in blue. COPII, COPII vesicles; $N$, nucleus; $L E$, late endosomes.

iments revealed that CLN2 protein interacts strongly with CLN3 compared with CLN6 and CLN8. It may be that the CLN2 forms a stronger association with CLN3 than with either CLN6 or CLN8 in vitro; or, more likely, that the antibodies to CLN2 and CLN3 were more specific than CLN6 or CLN8 antibody; or that protein levels of CLN6 and CLN8 are lower than CLN2 and CLN3 protein levels. Expression levels of CLN6 and CLN8 may be too low for detection before immunoprecipitation and/or transfection.

Interacting proteins may co-localize in the same subcellular locations. CLN2, CLN3, CLN6, and CLN8 proteins colocalized in a number of subcellular compartments. CLN3 was present in Golgi and Rab4- and Rab11-positive recycling endosomes and lipid rafts (4); CLN2 was in lysosomes and also in Rab11-positive recycling endosomes (Fig. 4). This may contribute to the high-intensity bands observed in coimmunoprecipitation experiments. The co-localization distribution patterns for CLN3 and CLN2, suggest that CLN3 and CLN2 primarily co-localize within Rab11-positive recycling endosomes and lipid rafts.

CLN6 and CLN8 proteins are present within ER. Additionally, we determined CLN6 protein presence within Rab4positive endosomes and lipid rafts, and CLN8 protein presence in lipid rafts. Co-localization of CLN6 and CLN2 occurred within lipid rafts. Co-localization patterns of CLN6 and CLN8 appeared vesicular suggesting presence in recycling compartments, as suggested for CLN8p or in lipid rafts. Co-localization patterns of CLN3 and CLN6, and CLN3 and CLN8 were similar, showing overlap in lipid rafts at the plasma membrane and in recycling endosomes. CLN6 and CLN8 co-localized to a greater degree within vesicular structures than to lipid rafts, suggesting CLN8 spends little time in lipid rafts.

Surprising to us was the degree of co-localization between the CLN proteins and different organelles. Looking at multiple $\mathrm{Z}$ planes in each cell led to determination of which plane showed maximal co-localization. It was difficult to distinguish between different vesicular compartments as they are in proximity to each other, with constant flow of cargo. It is suggested that these proteins are being recycled between subcellular 
compartments and lipid rafts at the plasma membrane. Defects in any of these NCL proteins may result in abnormal trafficking and subsequent disease.

CLN1, CLN2, CLN3, and CLN8 have all been linked to sphingolipid metabolism. In INCL, there is lysosomal accumulation of sphingolipid activator proteins (SAP), and a decrease in phospholipids but increased phosphatidylcholine (PC), suggesting disruption in the synthesis/trafficking of sphingolipids. In LINCL patients, the distribution of ethanolamine plasmalogens (or alkenyl acyl GPE) species differed from healthy controls (19-21). Elevated levels of ceramide/ sphingomyelin characterizes CLN3-deficient fibroblasts. CLN3 protein restored these lipids to normal. Furthermore, CLN3 may be involved in the trafficking of GalCer between Golgi and lipid rafts (4). The EPMR/CLN8-deficient brain from an older patient showed altered levels of phospholipids/ sphingolipids. In particular, ceramide, galactosyl-/ lactosylceramide and sulfatide were reduced, while phosphatidylserine, phosphatidylethanolamine and phosphatidylinositol levels were increased. This suggests the involvement of altered membrane stability, and vesicular trafficking in the molecular pathogenesis of EPMR (22). Cumulatively, these studies lend credence to the hypothesis that the NCL proteins interact with each other, possibly through a common pathway that may involve sphingolipid metabolism.

There are multiple points at which the NCL proteins may interact (Fig. 7). From ER, these proteins may traffic to Golgi via COPII-positive vesicles. The CLN2 and CLN3 proteins may interact within Golgi, early/recycling endosomes, and lipid rafts. CLN6 and CLN8 proteins are also recycled via the endosomal machinery and at some point appear within lipid rafts. It appears that CLN1 may be downstream of the other NCL proteins addressed here. We may speculate that its distribution may be limited, with primary residence in lysosomes, where it can interact with CLN2. We provide evidence for the provocative idea that NCL proteins are essential components of a common pathway and that they may interact at multiple locations. This pathway may involve synthesis and/or trafficking of sphingolipids. Disruption of this pathway may lead to trafficking defects. This hypothesis is supported by previous work, which demonstrates the presence of a GalCer trafficking defect in JNCL. Better delineation of this common pathway and the molecular specifics of CLN protein interac- tions is necessary for developing therapeutic strategies to combat these deadly diseases.

\section{REFERENCES}

1. Boustany RM, Zucker A 2006 Diseases primarily of grey matter. In: Swaiman K, Ashwal S, Ferreiro D (eds) Pediatric Neurology: Principles and Practice. MosbyElsevier, Philadelphia

2. Persaud-Sawin DA, VanDongen A, Boustany RM 2002 Motifs within the CLN3 protein: modulation of cell growth rates and apoptosis. Hum Mol Genet 11:21292142

3. Rylova SN, Amalfitano A, Persaud-Sawin DA, Guo WX, Chang J, Jansen PJ, Proia $\mathrm{AD}$, Boustany RM 2002 The CLN3 gene is a novel molecular target for cancer drug discovery. Cancer Res 62:801-808

4. Persaud-Sawin DA, McNamara JO II, Rylova S, Vandongen A, Boustany RM 2004 A galactosylceramide binding domain is involved in trafficking of CLN3 from Golgi to rafts via recycling endosomes. Pediatr Res 56:449-463

5. Boustany RM 1992 Neurology of the neuronal ceroid-lipofuscinoses: late infantile and juvenile types. Am J Med Genet 42:533-535

6. Boustany RM, Alroy J, Kolodny EH 1988 Clinical classification of neuronal ceroid-lipofuscinosis subtypes. Am J Med Genet Suppl 5:47-58

7. Boustany RM, Filipek P 1993 Seizures, depression and dementia in teenagers with Batten disease. J Inherit Metab Dis 16:252-255

8. Goebel HH 1995 The neuronal ceroid-lipofuscinoses. J Child Neurol 10:424-437

9. Goebel HH 1996 The neuronal ceroid-lipofuscinoses. Semin Pediatr Neurol 3:270 278

10. Goebel HH, Schochet SS, Jaynes M, Bruck W, Kohlschutter A, Hentati F 1999 Progress in neuropathology of the neuronal ceroid lipofuscinoses. Mol Genet Metab 66:367-372

11. Goebel HH, Sharp JD 1998 The neuronal ceroid-lipofuscinoses. Recent advances. Brain Pathol 8:151-162

12. Santavuori P, Heiskala H, Autti T, Johansson E, Westermarck T 1989 Comparison of the clinical courses in patients with juvenile neuronal ceroid lipofuscinosis receiving antioxidant treatment and those without antioxidant treatment. Adv Exp Med Biol 266:273-282

13. Santavuori P, Vanhanen SL, Autti T 2001 Clinical and neuroradiological diagnostic aspects of neuronal ceroid lipofuscinoses disorders. Eur J Paediatr Neurol 5(suppl A):157-161

14. Sleat DE, Sohar I, Pullarkat PS, Lobel P, Pullarkat RK 1998 Specific alterations in levels of mannose 6-phosphorylated glycoproteins in different neuronal ceroid lipofuscinoses. Biochem J 334:547-551

15. Weimer JM, Kriscenski-Perry E, Elshatory Y, Pearce DA 2002 The neuronal ceroid lipofuscinoses: mutations in different proteins result in similar disease. Neuromolecular Med 1:111-124

16. Rossetti S, Hoogeveen AT, Sacchi N 2004 The MTG proteins: chromatin repression players with a passion for networking. Genomics 84:1-9

17. Bratton SB, MacFarlane M, Cain K, Cohen GM 2000 Protein complexes activate distinct caspase cascades in death receptor and stress-induced apoptosis. Exp Cell Res 256:27-33

18. Vesa J, Chin MH, Oelgeschlager K, Isosomppi J, DellAngelica EC, Jalanko A, Peltonen L 2002 Neuronal ceroid lipofuscinoses are connected at molecular level: interaction of CLN5 protein with CLN2 and CLN3. Mol Biol Cell 13:2410-2420

19. Haltia M, Tyynela J, Baumann M, Henseler M, Sandhoff K 1995 Immunological studies on sphingolipid activator proteins in the neuronal ceroid-lipofuscinoses. Gerontology 41:239-248

20. Granier LA, Langley K, Leray C, Sarlieve LL 2000 Phospholipid composition in late infantile neuronal ceroid lipofuscinosis. Eur J Clin Invest 30:1011-1017

21. Tyynela J, Suopanki J, Baumann M, Haltia M 1997 Sphingolipid activator proteins (SAPs) in neuronal ceroid lipofuscinoses (NCL). Neuropediatrics 28:45-48

22. Hermansson M, Kakela R, Berghall M, Lehesjoki AE, Somerharju P, Lahtinen U 2005 Mass spectrometric analysis reveals changes in phospholipid, neutral sphingolipid and sulfatide molecular species in progressive epilepsy with mental retardation, EPMR, brain: a case study. J Neurochem 95:609-617 